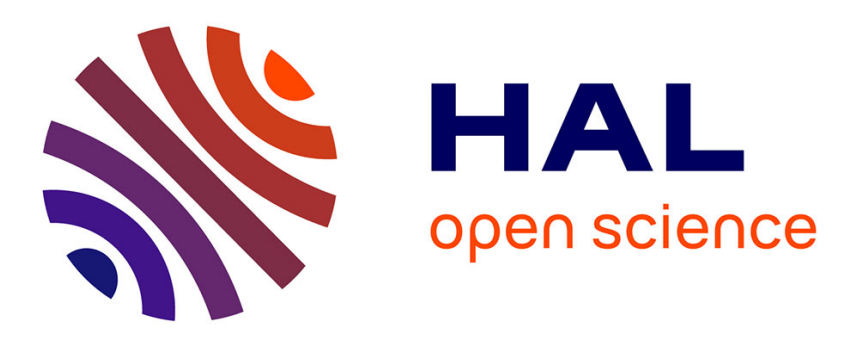

\title{
A systematic typology for negative Poisson's ratio materials and the prediction of complete auxeticity in pure silica zeolite JST
}

\author{
M. Siddorn, François-Xavier Coudert, K. E Evans, A. Marmier
}

\section{- To cite this version:}

M. Siddorn, François-Xavier Coudert, K. E Evans, A. Marmier. A systematic typology for negative Poisson's ratio materials and the prediction of complete auxeticity in pure silica zeolite JST. Physical Chemistry Chemical Physics, 2015, 17 (27), pp.17927-17933. 10.1039/C5CP01168J . hal-02190936

\author{
HAL Id: hal-02190936 \\ https://hal.science/hal-02190936
}

Submitted on 23 Jul 2019

HAL is a multi-disciplinary open access archive for the deposit and dissemination of scientific research documents, whether they are published or not. The documents may come from teaching and research institutions in France or abroad, or from public or private research centers.
L'archive ouverte pluridisciplinaire HAL, est destinée au dépôt et à la diffusion de documents scientifiques de niveau recherche, publiés ou non, émanant des établissements d'enseignement et de recherche français ou étrangers, des laboratoires publics ou privés. 


\section{ARTICLE}

Cite this: DOI: $10.1039 /$ xoxxooooox

Received ooth January 2012

Accepted ooth January 2012

DOI: $10.1039 /$ xoxxooooox

www.rsc.org/PCCP

\section{A systematic typology for negative Poisson's ratio materials and the prediction of complete auxeticity in pure silica zeolite $\mathbf{J S T}^{\dagger}$}

\author{
M. Siddorn, ${ }^{a}$ F.-X. Coudert, ${ }^{b}$ K.E. Evans ${ }^{a}$ and A. Marmier ${ }^{a \neq}$
}

\begin{abstract}
Single crystals can commonly have negative Poisson's ratio in a few directions; however more generalised auxeticity is rarer. We propose a typology to distinguish auxetic materials. We characterise numerous single crystals and demonstrate that partial auxeticity occurs for around $37 \%$. We find average auxeticity to be limited to $\alpha$-cristobalite and no example of complete auxeticity. We simulate two hundreds pure silica zeolites with empirical potentials and quantum chemistry methods, and for the first time identify complete auxeticity in a zeolite network, JST.
\end{abstract}

\section{Introduction}

The main aims of this study are to develop a convenient typology of auxetic behaviour in materials, to characterise the Poisson's ratio of pure silica zeolites and to identify structures with exceptional values.

The Poisson's ratio for anisotropic materials is complex, a function of three variables, two defining a longitudinal direction, one a transverse one. The adjective "auxetic", describing the existence of a negative Poisson's ratio (NPR) ${ }^{1}$, is too limited to describe fundamentally different situations, from single crystals where NPR occurs for very specific directions, to isotropic foams where NPR is present for all directions. Therefore an important objective has been to develop a finergrained typology of auxetic properties to discriminate between the relatively common existence of negative Poisson's ratios in a few narrowly defined combinations of longitudinal and transverse directions and the rarer, more comprehensive cases.

\section{Background}

Of the four elastic constants used to describe isotropic materials, Young's modulus $(E)$, bulk modulus $(K)$, shear modulus $(G)$ and Poisson's ratio $(v)$, it is the Poisson's ratio that has historically been the least explored ${ }^{2,3}$. It can be associated with some interesting and unusual properties, particularly when in a range not normally encountered. Defined as the ratio of transverse to longitudinal strain in a structure or material, it has been accepted theory that the Poisson's ratio can have negative values for over 150 years but it is only since 1989 that it has been actively studied. Due to their unusual nature, auxetic materials could be used in many applications where the benefits of their properties have only recently become apparent. As Evans showed, highly negative $v(-1 / 2>v)$ can produce large values for indentation resistance and fracture toughness ${ }^{2}$. Of recent particular interest is the manufacture of blast curtains for defence purposes. When an object hits an auxetic fabric the surrounding material closes in on the impacted area rather than away from it, causing an increase in local density and potentially stopping fragments of shrapnel or projectiles ${ }^{4}$.

The motivation to study NPR in the family of silicate materials specifically is two-fold. Firstly, zeolites in particular have historically received considerable interest due to their very low density and potential use as catalysts ${ }^{5}$ or molecular sieves ${ }^{6,7}$. Early studies of their mechanical properties have been far from systematic and in light of recent results ${ }^{8,9}$ it is timely to revisit auxeticity in silicates in general, and zeolites in particular, to compare their mechanical properties with those of other crystalline materials. Secondly, it has been recognised that, singularly among single crystals, $\alpha$-cristobalite is auxetic in a large directions range ${ }^{10}$; a logical place to search for materials with equally exceptional properties is amongst other silicates, especially largely unexplored pure silica zeolites.

From Brillouin scattering on single crystals, the MFI zeolite was found to be auxetic in the (001) plane ${ }^{11}$. Auxetic zeolites are of interest due to the prospect that 'tuneable' molecular sieves could be created, for which the porosity could be altered by means of an applied stress ${ }^{12}$. Moreover, adsorption of specific guests in their nanopores could provide a way to tune their mechanical properties ${ }^{13}$. The negative Poisson's ratios observed in MFI and other zeolites has been explained using two dimensional mechanisms of rotating rigid bodies, and later more accurately using rotating semi-rigid bodies ${ }^{14}{ }^{15}$. Pure 
silica zeolites are difficult to synthetize as demonstrated by the fact that only 46 out of 206 known zeolitic frameworks have been experimentally synthesized as pure silica materials, but they provide a starting point for high-throughput modeling.

\section{Typology of auxetic materials}

Previous authors have realised that auxeticity can take different forms ${ }^{16,17}$ and introduced the concepts of partial auxeticity and complete auxeticity for cubic crystals. The scheme we propose is more detailed and not restricted to cubic crystals. A material which exhibits auxetic behaviour may experience a negative Poisson's ratio in a very narrow range of transverse directions, for a narrow range of longitudinal directions of deformation. Conversely, it could be isotropic and exhibit negative Poisson's ratios for all transverse directions, for every longitudinal deformation; or it could be anywhere in-between. In order to more accurately discuss auxetic materials and their nature it is therefore desirable to establish a typology in which all categories of auxetic materials can be identified, described and distinguished. The following section describes such a general typology of the auxetic behaviour of a material.

\subsection{Basic principles}

In this context, we use the term 'direction' to refer to the transverse direction for which Poisson's ratio is observed and 'axis of deformation' to refer to the longitudinal axis of loading. Our classification is based on a type, a class and a corresponding numerical indicator; Table 1 describes the 10 possible combinations, from 0 (non auxetic), to $3 \mathrm{C}$ (auxetic for all axes and directions). 'Type' describes the axes of deformation, it indicates whether there is NPR around none (type 0 ), at least one (type 1), an average of (type 2), or every axis of deformation (type 3 ). 'Class' refines that information by considering NPR in the transverse directions, for at least one (class A), for an average (class B) or for every direction (class C).

Table 1 . Typology of auxetic materials

\begin{tabular}{cc}
\hline Code & Poisson's ratio is negative \\
\hline 0 & never \\
\hline 1A & for at least one direction around at least one axis \\
1B & averaged over all directions around at least one axis \\
1C & for all directions around at least one axis \\
\hline 2A & for at least one direction around an average of axes \\
2B & averaged over all directions around an average of axes \\
2C & for all directions around an average of axes \\
\hline 3A & for at least one direction around all axes \\
3B & averaged over all directions around all axes \\
3C & for all directions around all axes \\
\hline
\end{tabular}

This is moderately abstract, and to illustrate the criteria which must be satisfied for a material to fall into the respective categories, Fig. 1 shows the elastic transverse deformations around specific axes in four examples (in other words, the axis of deformation is perpendicular to the page). The plots (b), (c), and (d) in Fig. 1 give examples of materials which meet the minimum requirement to be classified as auxetic type $1 \mathrm{~A}, 1 \mathrm{~B}$ and $1 \mathrm{C}$ respectively. While it is enough for a material to respect one of these three criteria along one axis of deformation only to be auxetic type 1 , in order to be classified as type 2 the property must be present along an average of all axes of deformation (the way in which we decide if this average condition is met is a matter of choice and is discussed in more detail later in this section). To be classified as type 3 a material must meet the class criteria, shown in Fig. 1, for every axis of deformation. Such extreme auxeticity is rare in anisotropic materials but may be found in isotropic foams.

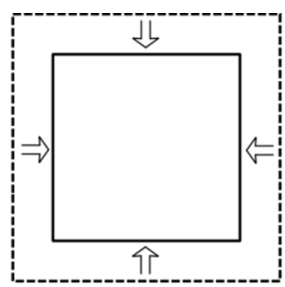

a)

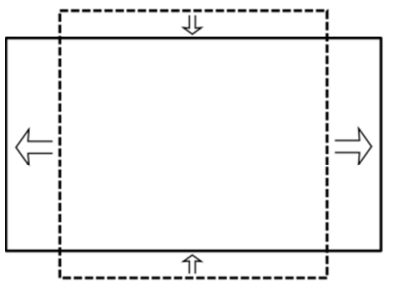

c)

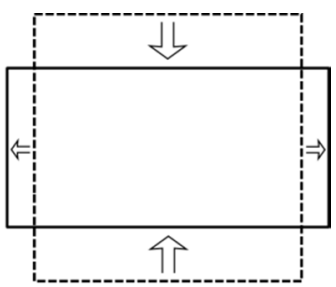

b)

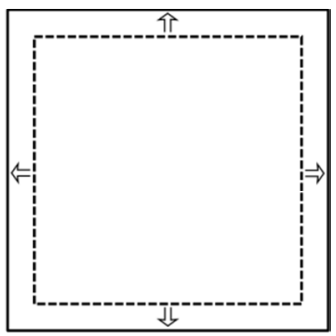

d)
Fig. 1. Cross section of materials of various auxetic typologies subjected to a tensile load in the direction normal to the page. The dotted line represents the original cross section, and the full line the final cross section. a) Conventional behaviour with only positive Poisson's ratio. b) The material meets the minimum requirements to be class $1 \mathrm{~A}$ as this axis has at least some NPR $\mathrm{c}$ ) The material meets the minimum requirements to be class $1 B$ as the mean of the Poisson's ratios around this axis is negative. d) The material meets the minimum requirements to be class $1 \mathrm{C}$ as $\mathrm{v}$ is negative for all transverse directions.

It should be pointed out that a material that falls into a particular category may also fall into one or more others. For example, it is entirely possible for a material to be both $2 \mathrm{~B}$ and 3A simultaneously. At the simpler level, lower classifications (that is to say less auxetic) are explicitly implied by all higher classifications. Both the type (1, 2 or 3$)$, and the class (A, B or $\mathrm{C})$, carry these implications, provided the alternate value does not increase. Consequently, if a material is $2 \mathrm{C}$ it follows it must also be $1 \mathrm{C}$, a classification with a lower type, and additionally $2 \mathrm{~B}$ and $2 \mathrm{~A}$, classifications with lower classes. A 2C material does not necessarily imply $3 \mathrm{~A}$, as although the class has been reduced, the type has been increase; a $3 \mathrm{~A}$ classification could still be satisfied, depending on the material.

When establishing the typology of an anisotropic material, the Poisson's ratio must be calculated for several directions around many axes. The Poisson's ratio is readily given by the opposite of the ratio of the two rotated elastic compliances, $S_{12}^{\prime}$ and $S_{11}^{\prime}$. The rotated compliances can themselves be derived from a set of elastic constants in the principle axes, be they in the form of the compliances matrix $S$, or more commonly the stiffness matrix $C$ (for more detail on the notations of elasticity, 
including relationship between order 4 tensors and order 2 matrices, see for instance ${ }^{18}$ or ${ }^{19}$ ). The elastic tensor/matrices have either been measured experimentally by methods such as Brillouin scattering or acoustic microscopy, or been calculated from the second derivatives of potential energy models, usually numerically, following an optimisation stage.

Calculating the Poisson's ratio for off-axis combination of longitudinal axis and transverse direction is not especially complex, but often tedious and repetitive. The method has been systematically implemented in the ElAM software package ${ }^{19}$. For a typical calculation, the three angles $(\theta, \varphi, \chi)$ describing the axis vector $\boldsymbol{a}$ and direction vector $\boldsymbol{b}$ each varies every degree (see Fig. 2). This generates an excess of 23 million sets of off-axis elastic constants, from which the extrema and averages necessary to establish the auxetic typology can be determined.

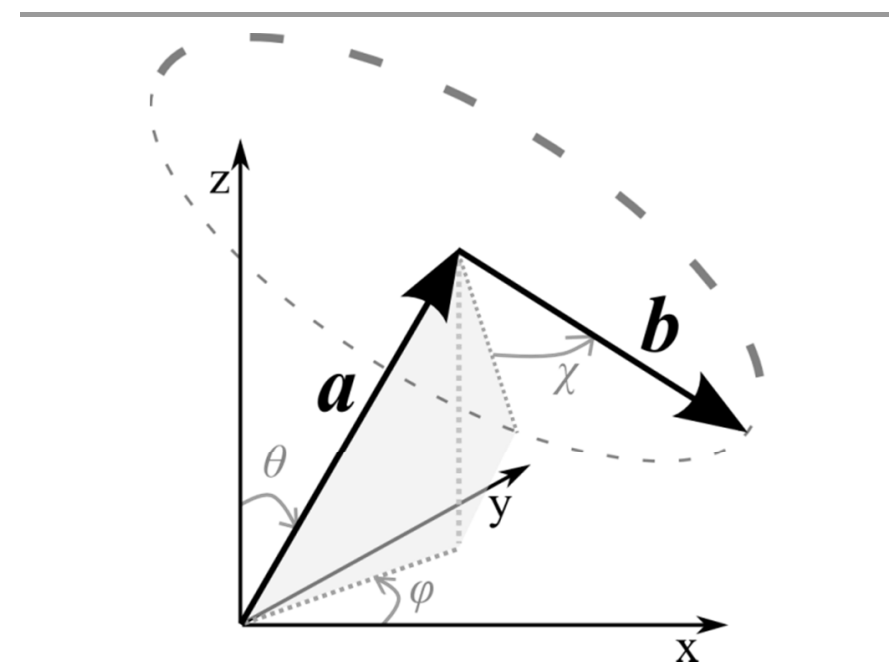

Fig. 2. Angles and vectors describing longitudinal axis $(a)$ and transverse direction (b)

\subsection{Additional considerations on averages}

Class A and class $\mathrm{C}$ can be determined unambiguously by the minimum or maximum $v$ around a single axis (if maximum $v$ is negative then class $\mathrm{C}$, if minimum $v$ is negative then class A). Class $\mathrm{B}$ relies on $v$ being averaged, and different options for this are possible. The average could be considered as a direct mean (if the mean $v$ is negative then class B is satisfied), or a simpler median average (if more than half of the $v$ values are negative then class $B$ is satisfied). When many single crystals are arranged in a random orientation, it is the average of the Poisson's ratio values that will affect the polycrystalline properties ${ }^{20}$ rather than the total number of direction exhibiting a particular Poisson's ratio in each single crystal. Therefore, in this paper the mean Poisson's ratio is used to ascertain if a material satisfies class $\mathrm{B}$, as this will give a greater idea as to which are likely to exhibit auxetic behaviour in a polycrystalline structure.

The type section of a classification is derived from the number of axes which exhibit the three different classes. The more generally a class is exhibited, the higher the type of this class will be. Type 1 , the simplest case, is satisfied if there is any behaviour of a class exhibited by the material. Therefore, if at least one axis has a particular class then type 1 of this class will be satisfied.

In order for a material to satisfy type 2 classifications, what is considered as the 'average axis' must meet the criteria of a class. As the average needed is of classes, rather than simple numerical values, the decision process is more subtle than that for determining class B auxeticity and it is valid to consider two distinct criteria. The first of these is whether or not a randomly chosen axis is expected to be of a certain class. This can be expressed as the median of the class when considering all axes, where the classification will be satisfied if more than half of the axes examined exhibit a class. The second criteria considered deals with numerical values which directly relate to the satisfaction of the three classes. Classes A, B, and C are satisfied when the minimum, average, and maximum Poisson's ratios are negative respectively. A material can be considered to be type 2 if the average of these numerical values for all axes is also negative.

With this in mind, the classification for type 2 is split into two separate variants: $2 \mathrm{i}$ and $2 \mathrm{ii}$. Type $2 \mathrm{i}$ is satisfied if more than half of the axes examined show behaviour of a particular class. Type $2 \mathrm{ii}$ is satisfied if the mean of relevant values to the class are negative, so that a material is $2 \mathrm{iiA}$ if the mean of the minimum $v$ is negative, $2 \mathrm{iiB}$ if the mean of the mean $v$ is negative, and $2 \mathrm{iiC}$ if the mean of the maximum $v$ is negative.

Type 3 is satisfied if a material exhibits a class completely, when any axis of deformation is considered. Therefore, if every axis examined is of a particular class then type 3 of this class will be satisfied for the material.

To allow comparisons between materials and enable us to identify materials which may be of interest, each classification in this typology is matched with a value relevant to the criteria used to establish its satisfaction. Table 2 details the values which accompany each classification of the auxetic behaviour of a system.

\begin{tabular}{cc}
\hline Table 2. Auxetic typology values \\
\hline \multicolumn{3}{c}{ Code } & Value \\
\hline 0 & N/A \\
\hline $1 \mathrm{~A}$ & Minimum of the minimum $v$ \\
$1 \mathrm{~B}$ & Minimum of the average $v$ \\
$1 \mathrm{C}$ & Minimum of the maximum $v$ \\
\hline $2 \mathrm{iA}$ & Percentage of axes of class A \\
$2 \mathrm{iB}$ & Percentage of axes of class B \\
$2 \mathrm{iC}$ & Percentage of axes of class C \\
\hline $2 \mathrm{iiA}$ & Average of the minimum $v$ \\
$2 \mathrm{iiB}$ & Average of the average $v$ \\
$2 \mathrm{iiC}$ & Average of the maximum $v$ \\
\hline $3 \mathrm{~A}$ & Maximum of the minimum $v$ \\
$3 \mathrm{~B}$ & Maximum of the average $v$ \\
$3 \mathrm{C}$ & Maximum of the maximum $v$ \\
\hline
\end{tabular}

Of particular interest are materials which meet the criteria for satisfying 2iiB. Because these materials show a negative Poisson's ratio when averaged over all axes and all directions, 
they should exhibit isotropic auxetic behaviour when part of randomly arranged polycrystalline structures.

\subsection{Case Study for general materials}

To demonstrate the effectiveness of the classification system when used as a comparison criterion between different groups of materials and to establish a baseline of auxeticity, we first study a wide range of different materials. The experimental elastic constants of the same 471 materials used in the work on the correlation between extreme Poisson's ratios and anisotropy by Lethbridge ${ }^{8}$ are used to derive auxetic typologies, as they are considered to be representative of the auxetic properties of single crystals in general.

Table 3. Auxetic classifications for case study on general materials

\begin{tabular}{ccc}
\hline Code & Number & Percentage \\
\hline 0 & 296 & 62.8 \\
\hline $1 \mathrm{~A}$ & 175 & 37.2 \\
$1 \mathrm{~B}$ & 15 & 3.2 \\
$1 \mathrm{C}$ & 11 & 2.3 \\
\hline $2 \mathrm{iA}$ & 31 & 6.6 \\
$2 \mathrm{iB}$ & 1 & 0.2 \\
$2 \mathrm{iC}$ & 1 & 0.2 \\
\hline $2 \mathrm{iiA}$ & 37 & 7.9 \\
$2 \mathrm{iiB}$ & 1 & 0.2 \\
$2 \mathrm{iiC}$ & 1 & 0.2 \\
\hline $3 \mathrm{~A}$ & 3 & 0.6 \\
$3 \mathrm{~B}$ & 0 & 0.0 \\
$3 \mathrm{C}$ & 0 & 0.0 \\
\hline
\end{tabular}

Table 3 shows the number of materials for each typology. Crystals exhibiting weak auxetic behaviour of type 1A are surprisingly common, $37.2 \%$ of those studied. However, the number of materials satisfying increasing levels of auxeticity decreases rapidly, with only $3.2 \% 1 \mathrm{~B}, 6.6 \% 2 \mathrm{iA}$, and $7.9 \%$ $2 \mathrm{iiA}$. The $2 \mathrm{iB}, 2 \mathrm{iiB}$ and $2 \mathrm{C}$ categories are populated by a single crystal, $\alpha$-cristobalite, with auxetic coverage of $99 \%$ (percentage of axes with negative average PR) -0.14 (average of average) and -0.002 (average of maximum). Three single crystals belong to the $3 \mathrm{~A}$ category: $\alpha$-cristobalite again, and two forms of Beryllium Copper alloy with auxetic values (maximum of the minimum) of $-0.063,-0.003$, and 0.0 respectively. None of the 471 single crystals has $3 \mathrm{~B}$ or $3 \mathrm{C}$ auxeticity. We do not observe any correlation between simpler, non-elastic tensor related properties and the presence or extent of auxeticity. In particular, and somewhat counterintuitively, crystal systems, space groups or even atomic structures are not predictors of auxeticity: for instance, $57 \%$ of the BCC metals are auxetic, but $43 \%$, with the same atomic structure, are not ${ }^{21}$.

\section{$3.4 \alpha$-Cristobalite}

As seen from this meta-analysis, the auxeticity of $\alpha$-cristobalite is very peculiar, not because of the low values its Poisson's ratio reaches $(-0.51$, quite modest), but because of the large range of directions for which it has a negative value.

The silicon dioxide polymorph cristobalite has two crystalline phases, a low temperature $(\alpha)$ form and a high temperature $(\beta)$ form. Brillouin spectroscopy on a single crystal of $\alpha$-cristobalite showed it to be highly anisotropic and yet still have an aggregate isotropic Poisson's ratio of $-0.133,-0.191$ and -0.163 for the Reuss, Voigt and Hill averages respectively ${ }^{10}$. This study also showed that the crystal had a shear modulus of roughly 2.4 times that of the bulk modulus. Due to the instability of $\beta$-cristobalite, its elastic properties have only been derived through the use of computational modelling. A molecular dynamics study showed that the high temperature phase of cristobalite remains averagely auxetic ${ }^{22}$. Structurally $\alpha$-cristobalite is analogous to a tetragon formed by four smaller tetrahedra which are able to rotate and dilate.

The unique auxetic nature of $\alpha$-cristobalite is highlighted by our classification system. Within the auxetic typology it satisfies all classifications except $3 \mathrm{~B}$ and $3 \mathrm{C}$. Considered in context as a member of the materials previously examined in this study, it is the only one of the 471 to exhibit $2 \mathrm{iB}, 2 \mathrm{iC}, 2 \mathrm{iiB}$, and $2 \mathrm{iiC}$ auxeticity and one of the three materials to satisfy type $3 \mathrm{~A}$. Through use of the classification system proposed $\alpha$-cristobalite is confirmed as extreme, even among other materials exhibiting a negative Poisson's ratio.

\section{Atomistic simulations of all-silica-zeolites}

\subsection{Methods}

In order to extend our sample of materials, we calculated the elastic properties of all-silica zeolites as well as $\alpha$-quartz and $\alpha$ cristobalite using classical and $a b$ initio simulations. Crystallographic information files (.cif) of the 206 known zeolite frameworks were obtained from the international zeolite association $^{23}$. The classical calculations were performed with GULP v3.1 ${ }^{24}$, using the five well established potential models of Catlow, Gale, Matsui, Sastre and Van Beest ${ }^{25-29}$. These models have different characteristics (partial/formal charges, shell models) and have been shown in a recent review to perform well for elastic properties of $\alpha$-quartz ${ }^{30}$. The first principles calculations were performed with the CRYSTAL09 $\operatorname{code}^{31}$ within the Density Functional Theory Theory (DFT) approach, using the B3LYP hybrid functional ${ }^{32}$ with empirical correction for the dispersive interactions ${ }^{33}$, full symmetry and all electron localized basis sets ${ }^{34}$. The stiffness matrixes for each structure ${ }^{\dagger}$ were used to build a database for the software ElAM $^{19}$ to examine. ElAM calculates the off axis elastic properties, allowing for the identification of interesting properties such as a negative Poisson's ratio in particular axes as well as establishing the auxetic typology. Not all pure silica frameworks could be relaxed. Depending on the potential models, between 186 and 189 were optimised with classical simulations. DFT simulations led to elastic constants for a subset of 121 frameworks, the remaining being too large for the computer resources available 9 . 


\subsection{Validation}

To ensure that the results are reasonable, it is critical to validate the models by comparison with experimental data. As little is available for the elastic constants of pure silica zeolites (we are only aware of $\mathrm{MFI}^{11}$ ), we have to rely on the elastic constants of the "dense" silicas $\alpha$-quartz and $\alpha$-cristobalite. Detailed comparisons with experimental lattice and elastic constants for $\alpha$-quartz, $\alpha$-cristobalite and MFI are available in tabulated form in table $\mathrm{S} 1(\mathrm{a}-\mathrm{c})^{\dagger}$. The root-mean-squared errors (RMSE) vary between $4 \mathrm{GPa}$ and $29 \mathrm{GPa}$ (average elastic constant at 40 GPa). DFT seems less accurate with RMSE at $29 \mathrm{GPa}$ (quartz) and $15 \mathrm{GPa}$ (cristobalite). The classical models fare equally well for quartz (4-16 GPa) and cristobalite (6-14 GPa). They are slightly less accurate for MFI (10-29 GPa), probably because the experiments were performed on crystals still containing the organic templates in their pores. The Catlow model is the most consistent with RMSE around $10 \mathrm{GPa}$ for all three crystals. It is notoriously difficult to simulate $\alpha$-quartz with DFT and elastic constants depend on precise details of the model. Another possible source of error is due to the fact that experimental elastic constants have been obtained at room temperature, while calculated ones are at $0 \mathrm{~K}$.

\section{$4.3 \alpha$-Cristobalite}

To further test the potential models used and to gain a better understanding of $\alpha$-cristobalite, the typology classifications for this crystal from differing potential models are compared. The elastic constants of $\alpha$-cristobalite are first calculated using the six potential models, resulting in six distinct stiffness matrices for comparison with experimental results. A typology classification is then created from each set of elastic data, and the associated values are shown in Table 4 , where the values which satisfy typology classifications are in bold. This table shows that subtle differences in elastic stiffness values strongly influence the classification, as $\alpha$-cristobalite does not reach ' $2 \mathrm{C}$ ' or ' $3 \mathrm{~A}$ ' for either of the potential models, and that the minimum Poisson's ratio (' $1 \mathrm{~A}$ ' value) is less by generally a third.
Table 4 - Associated values for the typology classifications of $\alpha$ cristobalite when using the elastic constants calculated with various potential models and from experimental results. Values corresponding to auxetic behaviour in bold (negative extremum or average above $50 \%$, see Table 2 ).

\begin{tabular}{cccccccc}
\hline \hline & Cat. & Gale & BKS & Sas. & Mat. & DFT & Exp. \\
\hline 1A & $\mathbf{- 0 . 3 2}$ & $\mathbf{- 0 . 3 5}$ & $\mathbf{- 0 . 4 6}$ & $\mathbf{- 0 . 3 4}$ & $\mathbf{- 0 . 4 7}$ & $\mathbf{- 0 . 4 1}$ & $\mathbf{- 0 . 5 1}$ \\
1B & $\mathbf{- 0 . 0 9}$ & $\mathbf{- 0 . 1 2}$ & $\mathbf{- 0 . 1 7}$ & $\mathbf{- 0 . 0 4}$ & $\mathbf{- 0 . 1 7}$ & $\mathbf{- 0 . 1 3}$ & $\mathbf{- 0 . 2 6}$ \\
1C & 0.05 & 0.04 & 0.02 & 0.02 & 0.01 & 0.04 & $\mathbf{- 0 . 0 7}$ \\
\hline 2iA & $\mathbf{5 9 \%}$ & $\mathbf{6 5 \%}$ & $\mathbf{8 3 \%}$ & $\mathbf{5 1 \%}$ & $\mathbf{9 0 \%}$ & $\mathbf{7 5 \%}$ & $\mathbf{1 0 0 \%}$ \\
2iB & $39 \%$ & $48 \%$ & $\mathbf{6 1 \%}$ & $8 \%$ & $\mathbf{6 6 \%}$ & $49 \%$ & $\mathbf{9 9 \%}$ \\
2iC & $0 \%$ & $4 \%$ & $0 \%$ & $0 \%$ & $0 \%$ & $0 \%$ & $\mathbf{5 3 \%}$ \\
\hline 2iiA & $\mathbf{- 0 . 0 9}$ & $\mathbf{- 0 . 1 1}$ & $\mathbf{- 0 . 1 9}$ & $\mathbf{- 0 . 0 1}$ & $\mathbf{- 0 . 2 0}$ & $\mathbf{- 0 . 1 5}$ & $\mathbf{- 0 . 2 8}$ \\
2iiB & 0.02 & $\mathbf{- 0 . 0 0}$ & $\mathbf{- 0 . 0 4}$ & 0.13 & $\mathbf{- 0 . 0 4}$ & 0.01 & $\mathbf{- 0 . 1 4}$ \\
2iiC & 0.13 & 0.10 & 0.12 & 0.27 & 0.13 & 0.16 & 0.00 \\
\hline 3A & 0.12 & 0.10 & 0.03 & 0.28 & 0.01 & 0.07 & $\mathbf{- 0 . 0 7}$ \\
3B & 0.12 & 0.10 & 0.13 & 0.28 & 0.13 & 0.21 & 0.00 \\
3C & 0.18 & 0.15 & 0.23 & 0.41 & 0.25 & 0.39 & 0.10 \\
\hline \hline
\end{tabular}

We conclude that used together, not in isolation, the six models provide a reasonable, if somewhat conservative, platform to explore the occurrence of NPR in pure silica zeolites.

\subsection{Auxetic typology of all silica zeolites}

Table 5 shows the percentages of pure silica zeolites that exhibit a certain type of auxeticity for all six models and contrasts them with the base line of materials established previously.

Table 5. Percentage of auxetic zeolites (from between 186 and 189 zeolites, depending on model) in each auxetic typology, compared with equivalent percentages for reference materials (471 single crystals)

\begin{tabular}{cccccccc}
\hline & Cat. & Gale & BKS & Sas. & Mat. & DFT & Ref. \\
\hline 0 & 67.4 & 83.4 & 51.3 & 78.6 & 54.0 & 74.4 & 62.8 \\
\hline $1 \mathrm{~A}$ & 32.6 & 16.6 & 48.7 & 21.4 & 46.0 & 25.6 & 37.2 \\
$1 \mathrm{~B}$ & 11.8 & 7.0 & 21.4 & 8.0 & 24.9 & 2.5 & 3.2 \\
$1 \mathrm{C}$ & 1.1 & 0.5 & 7.0 & 0.5 & 6.9 & 0.8 & 2.3 \\
\hline $2 \mathrm{iA}$ & 10.7 & 5.9 & 22.5 & 8.6 & 24.9 & 1.7 & 6.6 \\
$2 \mathrm{iB}$ & 1.0 & 0.5 & 2.7 & 0.5 & 1.1 & 0.8 & 0.2 \\
$2 \mathrm{iC}$ & 0.5 & 0.5 & 0.5 & 0.5 & 0.5 & 0.8 & 0.2 \\
\hline 2iiA & 12.8 & 6.4 & 25.1 & 8.6 & 27.5 & 0.8 & 7.9 \\
$2 \mathrm{iiB}$ & 1.6 & 1.0 & 1.6 & 1.6 & 0.5 & 0.8 & 0.2 \\
$2 \mathrm{iiC}$ & 0.5 & 0.5 & 0.5 & 0.5 & 0.5 & 0.8 & 0.2 \\
\hline 3A & 0.5 & 0.5 & 0.5 & 0.5 & 0.5 & 0.8 & 0.6 \\
3B & 0.5 & 0.5 & 0.5 & 0.5 & 0.5 & 0.8 & 0.0 \\
3C & 0.5 & 0.5 & 0.5 & 0.5 & 0.5 & 0.8 & 0.0 \\
\hline
\end{tabular}

Category 1A bears the most relevant comparison to previous studies, as it corresponds to a material exhibiting at least some negative Poisson's ratio. The proportions vary between $16.6 \%$ and $48.7 \%$, which correlate well with the $37.2 \%$ base line. They are significantly lower than the $69 \%$ of $1 \mathrm{~A}$ for cubic metals $^{21}$. One could be tempted to draw the counterintuitive conclusion that, as a class of materials, zeolites, despite their 
pores and low density are no more auxetic than normal materials. On closer inspections however, pure silica zeolites appear to have a significantly higher level of average auxeticity with increased percentages satisfying $2 \mathrm{iiA}$ and $1 \mathrm{~B}$ for most models. This somewhat vindicates the hypothesis that zeolites are good candidates for auxeticity, but barely. Following a trend similar to that of the reference sample, the proportions decrease rapidly above these classifications, with few materials displaying type 3 or class $\mathrm{C}$ auxeticity, with one notable exception, discussed later.

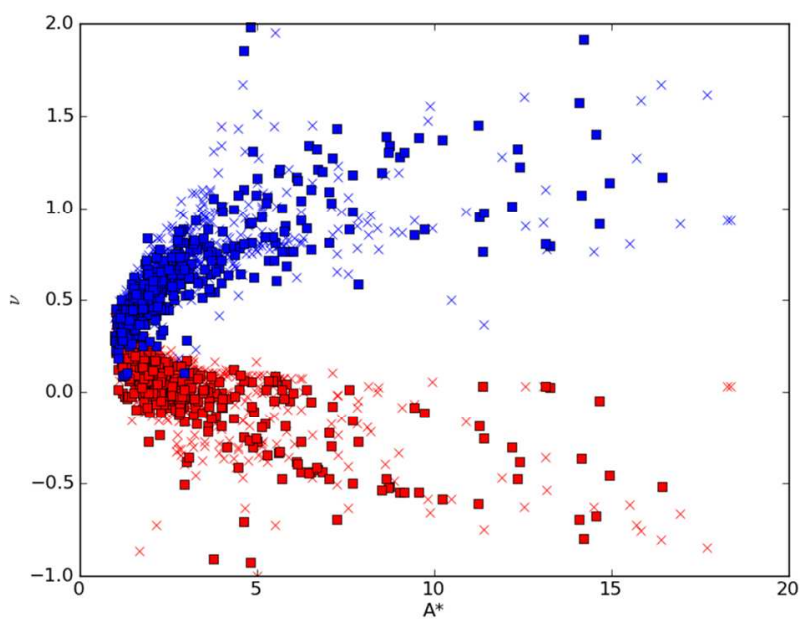

Fig. 3. Minimum and maximum Poisson's ratio vs Ledbetter Anisotropy for pure silica zeolites (crosses) and other materials (squares). Both $v$ and $A^{*}$ are unitless.

Comparison of the minimum and maximum calculated Poisson's ratio with elastic anisotropy confirms the work of Lethbridge et al. ${ }^{8}$ It was found that the extreme Poisson's ratio all lie on two families of curves approximately symmetrical around a single point of intersection at $A^{*}=1$, where $A^{*}$ is the Ledbetter anisotropy measure defined as the square of the maximum shear-sound-wave velocity divided by the square of the minimum shear-sound-wave velocity ${ }^{35}$. Fig. 3 shows the graph of minimum and maximum Poisson's ratio plotted against the elastic anisotropy of the structure, and the pure silica zeolites follow the same patterns as the sample of 471 single crystals. This suggests that overall, zeolite frameworks behave no differently than denser silica crystals.

A very small number of frameworks are 2B auxetic: with Catlow GOO, JST, NPO and VFI, with Gale JST and OSI, with Sastre ABW, IWV, JST and NPO, with BKS AFO, BOF, GON and JST, with Matsui MSO and JST, and with DFT JST only. Only one framework, JST, is 3C but crucially with all 6 models, including DFT.

\subsection{A completely auxetic zeolite: JST}

Table 6 Lattice parameter $a(\AA)$, elastic constants $C_{i j}(\mathrm{GPa})$, anisotropy index $A^{*}$ and auxeticity typology values $1 \mathrm{~A}, 2 \mathrm{~B}$ and $3 \mathrm{C}$ for JST

\begin{tabular}{ccccccc}
\hline & Cat. & Gale & Sas. & BKS & Mat. & DFT \\
\hline$a$ & 15.21 & 15.43 & 15.12 & 15.25 & 15.83 & 15.38 \\
\hline$C_{11}$ & 36.5 & 34.1 & 19.6 & 15.6 & 15.4 & 29.41 \\
$C_{12}$ & -5.7 & -6.28 & -7.7 & -13.1 & -8.8 & -8.4 \\
$C_{44}$ & 21.4 & 20.4 & 13.9 & 14.2 & 13.1 & 18.2 \\
\hline$A^{*}$ & 1.01 & 1.01 & 1.02 & 1.01 & 1.08 & 1.04 \\
\hline 1A & -0.19 & -0.23 & -0.66 & -5.42 & -1.33 & -0.40 \\
2B & -0.19 & -0.23 & -0.65 & -5.34 & -1.32 & -0.39 \\
3C & -0.19 & -0.22 & -0.65 & -5.26 & -1.31 & -0.38
\end{tabular}

Table 6 displays the lattice parameter, elastic coefficients and typology values for JST simulated with the 6 models. It is striking to note that all models are in very good agreement and predict complete auxeticity (3C) and almost complete elastic isotropy $\left(A^{*} \sim 1\right)$. On the other hand, the actual values for the Poisson's ratio, here in the form of auxeticity typology values, are less well defined, and vary from -0.2 to -5.4 . The most unusual feature of the elastic coefficients is that $\mathrm{C}_{12}$ is negative. While clearly uncommon, this has previously been observed experimentally by Brillouin scattering for $\mathrm{Sm}_{\mathrm{x}} \mathrm{La}_{1-\mathrm{x}} \mathrm{S}^{36}$. Negative $\mathrm{C}_{12}$ and sufficiently large $\mathrm{C}_{44}$ are necessary and sufficient conditions for a cubic crystal to be completely auxetic ${ }^{\dagger}$. Goldstein et al. list 6 possible completely auxetic cubic crystals (table S6 of the supporting material of ${ }^{17}$ ); it is interesting that 5 are samarium based, which strongly suggests a chemical bonding origin for their generalised auxeticity, as discussed in ${ }^{36}$.

In contrast, the remarkable agreement between the 6 models suggests that the completely auxetic character of JST derives chiefly from its structure, and not from details of the bonding. Pure silica JST is cubic (space group PA-3, number 205), its primitive cell, depicted in Fig. 4(a), contains 96 oxygen and 48 silicon. Due to this complexity it can be described in many ways. The following description is especially relevant to its auxeticity and helps understand the underlying mechanism. It is first useful to abstract the bent $\mathrm{Si}-\mathrm{O}-\mathrm{Si}$ bonds by straight $\mathrm{Si}-\mathrm{Si}$ segments as in Fig. 4(b). JST can then be seen as face centred cubic, where the motifs are four three-dimensional 6 sided stars. These stars (12 silicon each), are composed of a quasiplanar central hexagon surrounded by 6 sides alternatingly pointing up and down at angles of $\pm 60.4^{\circ}$ (in the Catlow model). The central hexagons lie in the four $\{111\}$ planes, and connect to each other through connecting triangles; in other words, each silicon belongs to a single star. If one assumes very stiff Si-Si connection, a tensile deformation flattens the stars, and makes them larger, leading to in-plane NPR. This simple mechanistic analysis is confirmed by studying the deformation of a unit cell. Under a $1 \%$ (100) strain, the $\mathrm{Si}-\mathrm{Si}$ bonds remain largely unchanged, most bonds deforming by $0.02 \%$ with a maximum of $0.18 \%$, while the out-of-plane side angle varies from $60.4^{\circ}$ to $58.9^{\circ}$, a change of $2.4 \%$. At this stage, JST has only been recently produced, and in a complex gallogermanate form with cationic templates in its pores ${ }^{37}$. While pure silica 
JST has not been synthesised yet, it might be necessary to obtain further confirmation of its framework's extraordinary auxeticity by mechanistic methods such as Finite Element Analysis or fabrication of macroscale models.

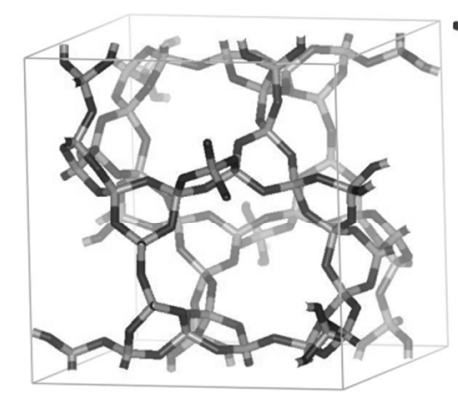

(a)

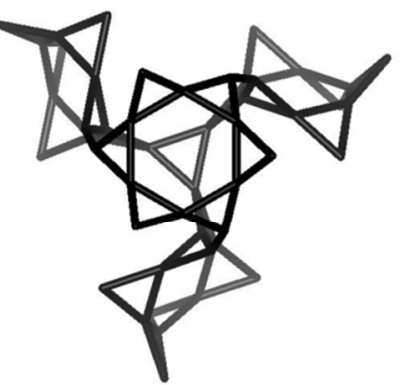

(b)
Fig. 4. (a) Unit cell of pure silica zeolite JST ( $\mathrm{Si}$ in light grey, $\mathrm{O}$ in dark); (b) Representation of JST, seen in the [111] direction. The vertices correspond to Si atoms, and the edges to Si-Si links.

We have designed a typology for auxetic materials, classifying materials with distinctions in the number of axes where a negative Poisson's ratio can be found, and the degree for which each axis is auxetic. The system has been demonstrated as a tool for comparing groups of materials and their auxetic properties, and the benefits of distinguishing between levels of auxeticity have also been explored, further highlighting the extraordinary properties of $\alpha$-cristobalite. The elastic properties of a large number of pure silica zeolite frameworks have been calculated and classified with the proposed typology. After comparison of the auxetic classifications with a database of 471 general materials, the pure silica zeolites are shown to be marginally more auxetic, but follow the same trends. We found that the JST frameworks has great potential for complete auxeticity, and have proposed an explanatory mechanism.

\section{Acknowledgements}

This work was supported by the Engineering and Physical Sciences Research Council [Grant number EP/G064601/1] ], and GENCI-IDRIS HPC resources [project i2014087069].

\section{Notes and references}

${ }^{a}$ College of Engineering, Mathematics and Physical Science, University of Exeter, EX4 4QF, UK.

${ }^{b}$ PSL Research University, Chimie ParisTech - CNRS, Institut de Recherche de Chimie Paris, 75005, Paris, France

$\dagger$ Electronic Supplementary Information (ESI) available: Detailed exposition of the auxetic typology and elastic constants for all calculated zeolites. See DOI: 10.1039/c000000x/

\$ Corresponding author: a.s.h.marmier@exeter.ac.uk

1. K. E. Evans, M. A. Nkansah, I. J. Hutchinson and S. C. Rogers, Nature, 1991, 353, 124-124.

2. K. E. Evans and A. Alderson, Adv. Mater., 2000, 12, 617-+.
3

4.

5.

6.

7.

8.

9.

10.

11.

12.

13.

14.

15

15.

16.

17.

18.

19.

20.

21.

22.

23.

$$
24 .
$$

25.

26.

27.

28.

29.

30.

31.

\section{2}

33.

34.

35.

36.

37.
G. N. Greaves, A. Greer, R. Lakes and T. Rouxel, Nature materials, 2011, 10, 823-837.

, US Patent 20110209557, 2010.

S. Kesraoui-Ouki, C. R. Cheeseman and R. Perry, J. Chem. Technol. Biotechnol., 1994, 59, 121-126.

A. Alderson, P. Davies, M. Williams, K. Evans, K. Alderson and J. Grima, Molecular Simulation, 2005, 31, 897-905.

M. E. Davis and R. F. Lobo, Chem. Mater., 1992, 4, 756-768.

Z. A. Lethbridge, R. I. Walton, A. S. Marmier, C. W. Smith and K. E. Evans, Acta Mater., 2010, 58, 6444-6451.

F.-X. Coudert, PCCP, 2013, 15, 16012-16018.

A. Yeganeh-Haeri, D. J. Weidner and J. B. Parise, Science, 1992, 257, 650-652.

C. Sanchez-Valle, Z. A. Lethbridge, S. V. Sinogeikin, J. J. Williams, R. I. Walton, K. E. Evans and J. D. Bass, J. Chem. Phys., 2008, 128, 184503-184503.

J. N. Grima, R. Gatt, V. Zammit, J. J. Williams, K. E. Evans, A Alderson and R. I. Walton, J. Appl. Phys., 2007, 101, 086102.

B. Coasne, J. Haines, C. Levelut, O. Cambon, M. Santoro, F. Gorelli and G. Garbarino, PCCP, 2011, 13, 20096-20099.

J. Grima, A. Alderson and K. Evans, Phys. Status Solidi B, 2005, 242, 561-575.

J. Grima, V. Zammit, R. Gatt, A. Alderson and K. Evans, Phys. Status Solidi B, 2007, 244, 866-882.

A. Brańka, D. Heyes and K. Wojciechowski, Phys. Status Solidi $B, 2009,246,2063-2071$.

R. V. Goldstein, V. A. Gorodtsov and D. S. Lisovenko, Phys. Status Solidi B, 2013, 250, 2038-2043.

J. F. Nye, Physical properties of crystals, Clarendon press, Oxford, 1985.

A. Marmier, Z. A. D. Lethbridge, R. I. Walton, C. W. Smith, S. C. Parker and K. E. Evans, Comput. Phys. Commun., 2010, 181, 2102-2115.

J. P. Watt, G. F. Davies and R. J. O'Connell, Rev. Geophys., 1976, 14, 541-563.

R. H. Baughman, J. M. Shacklette, A. A. Zakhidov and S. Stafstrom, Nature, 1998, 392, 362-365.

H. Kimizuka, H. Kaburaki and Y. Kogure, Phys. Rev. Lett., 2000, 84, 5548-5551.

C. Baerlocher and M. B. McCusker, in Database of zeolite structures: http://iza-structure.org/databases/.

J. D. Gale and A. L. Rohl, Molecular Simulation, 2003, 29, 291341 .

M. Sanders, M. Leslie and C. Catlow, J. Chem. Soc., Chem. Commun., 1984, 19, 1271-1273.

J. D. Gale, J. Phys. Chem. B, 1998, 102, 5423-5431.

S. Tsuneyuki, M. Tsukada, H. Aoki and Y. Matsui, Phys. Rev. Lett., 1988, 61, 869-872.

G. Sastre and J. D. Gale, Chem. Mater., 2003, 15, 1788-1796.

B. Van Beest, G. J. Kramer and R. Van Santen, Phys. Rev. Lett., 1990, 64, 1955.

A. F. Combariza, D. A. Gomez and G. Sastre, Chem. Soc. Rev., 2013, 42, 114-127.

R. Dovesi, R. Orlando, B. Civalleri, C. Roetti, V. R. Saunders and C. M. Zicovich-Wilson, Zeitschrift fur Kristallographie, 2005, 220, 571-573.

A. D. Becke, J. Chem. Phys., 1993, 98, 5648-5652.

S. Grimme, J. Comput. Chem., 2006, 27, 1787-1799.

R. Nada, J. B. Nicholas, M. I. McCarthy and A. C. Hess, Int. J. Quantum Chem., 1996, 60, 809-820.

H. Ledbetter and A. Migliori, Journal of Applied Physics, 2006, 100.

U. Schärer and P. Wachter, Solid State Commun., 1995, 96, 497501.

Y. Han, Y. Li, J. Yu and R. Xu, Angew. Chem. Int. Ed., 2011, 50, 3003-3005. 